\title{
Cost Design for Opportunistic Multi-Hop Routing in Cognitive Radio Networks
}

\author{
Miao Pan, Rongsheng Huang and Yuguang Fang \\ Department of Electrical and Computer Engineering \\ University of Florida, Gainesville, FL 32611 \\ Email: \{miaopan@, rshuang@, fang@ece.\}ufl.edu
}

\begin{abstract}
Cognitive Radio (CR) is a revolutionary technology with promising applications in military areas since it enables CR users in the field to dynamically access to the vacant licensed frequency bands if no primary users are present. In practice, multi-hop routing in $\mathrm{CR}$ networks presents a great challenge due to unreliable traditional links and time varying unlicensed CR links. To improve the performance of multi-hop routing, opportunistic routing (OR) has been proposed and investigated extensively. Instead of using a single next hop, $O R$ forwards a packet to an ordered set of candidate nodes and one node is chosen to relay the packet towards the destination. Most OR protocols prioritize the candidates and make the selection based on the cost defined as expected transmission times (ETX). Actually, ETX, as well as other existing criteria, does not always lead to the best forwarder choice for OR in CR networks since it ignores numerous potential CR links. In this paper, we propose a novel cost criterion for opportunistic multi-hop routing in CR networks, which leverages the unlicensed CR links to prioritize the candidate nodes and optimally selecting the forwarder. Simulation results show that our design efficiently decreases the number of transmissions, and effectively increases the throughput for most node pairs when compared with $O R$ and traditional single-path routing.
\end{abstract}

\section{INTRODUCTION}

Cognitive radio (CR) has received increasing attention in the past few years as a promising technology to implement the dynamic spectrum access and improve the

This work was supported by the National Science Foundation under grants CNS-0721744 and DBI-0529012. spectral efficiency [1]-[3]. Since CR users have the capability to sense, access to, and transmit on the licensed frequency bands opportunistically when the primary user is inactive [4], [5], the potential usage of CR networks has been recognized in broad areas, ranging from tactical communications in a battlefield, military sensing and tracking [6], to disaster rescue after the earthquake, public safety monitoring [7], etc.

Routing in multi-hop CR networks presents a great challenge mainly due to the following two facts. First, the traditional wireless links are not reliable because of channel fading [8]-[10]. Second, the validity of the CR links tends to be time varying and unpredictable because the communication between CR users depends on the activities of the primary user.

Traditional routing protocols have followed the routing concept in wired networks by abstracting wireless links as wired links, and endeavored to seek a fixed shortest path to forward packets between a pair of nodes. However, this abstraction ignores the broadcast nature and spacial diversity of the wireless medium. Taking advantage of these wireless natures, a new routing paradigm, known as opportunistic routing (OR) [11], has recently proposed to cope with the unreliability of link quality.

The basic idea behind OR is to integrate the network and media access control (MAC) layers such that at the network layer a set of forwarding candidates are selected and at the MAC layer one node is chosen as the actual relay. Owing to the broadcast nature and 
spatial diversity of the wireless medium, the probability of at least one forwarding candidate correctly receiving the packet will increase when multiple candidates are involved, thus improve the packet delivery efficiency such as throughput [12] or energy efficiency [13].

One important issue for realizing OR in CR networks is relay priority assignment, which has crucial impact on the performance of routing in CR networks, such as number of transmissions, end-to-end throughput, etc. Most existing works on OR [14], [15] leverage the expected transmission times (ETX) as the cost criterion to prioritize forwarding candidates. However, ignoring the opportunity to use potential CR links in the possible routes, these EXT based schemes do not always lead to the "best" forwarder selection.

In this paper, we are dedicated to designing a novel cost design for OR in CR network to specify the candidate forwarding list based on the characteristics of CR networks. Different from the multichannel and multi-rate OR schemes [8], CR not only provides backup channel but also offers more choices, e.g. supporting concurrent transmissions over different frequencies. Moreover, the link state may change quickly depending on the presence of the primary user. A CR based OR (CROR) cost criterion is proposed and four main contributions are made. Firstly, we develop a simple CR network example and identify the forwarder prioritization problems of ETX based OR to give some intuitive ideas. Secondly, we introduce the model of OR in CR network and formulate the activities of the primary user as well as the detection ability of the CR nodes. Thirdly, we design a cost criterion, CROR, to prioritize the candidate forwarders in OR considering the states of the CR links. Finally, we do simulations to evaluate the performance of the proposed CROR, and compare it with that of ETX based ExOR and LQSR ${ }^{1}$ [16].

Remainder of this paper is organized as follows. Section 2 describes the intuition behind our work, section 3 introduces the related concepts and models both in OR

\footnotetext{
${ }^{1}$ LQSR joins Dijkstra's algorithm with source routing, in which a source node puts the entire path of a flow in its packet headers and intermediate nodes forward the packets accordingly.
}

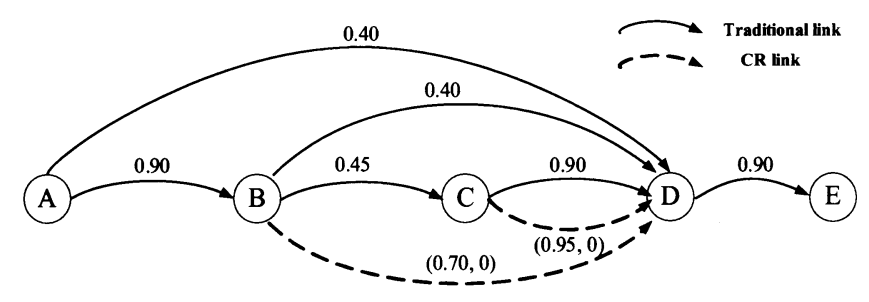

(a) The CR network with packet delivery ratios.

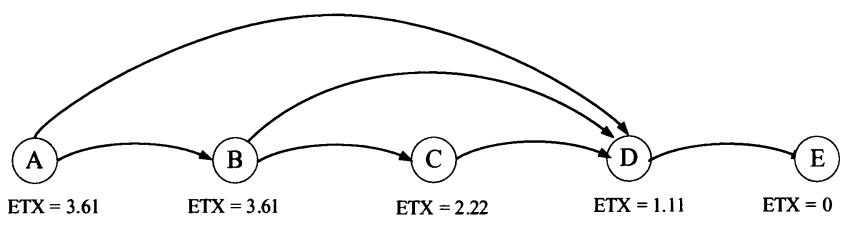

(b) Five node CR network with ETX

Fig. 1. Simple CR network example.

and CR network, section 4 elaborates on the details of the cost design, and section 5 compares the performance of OR based on proposed cost criterion with ETX based ExOR and LQSR in simulations. Finally, section 5 summarizes the remarks in the conclusion.

\section{INTUITION}

In order to develop an intuition for why there might be necessary for cost design of Opportunistic multi-hop routing in CR networks, it is helpful to consider the simple CR network in Fig. 1(a), in which there are a number of possible routes from source node $A$ to destination node $E$. Obviously, $A$ has two neighbors and must select a forwarder that may be used to reach the destination.

Assuming the complete set of inter-node packet delivery rate is known to the source as shown over the solid lines in Fig. 1(a), source node $A$ can leverage the knowledge to calculate all ETX cost. Fig. 1(b) shows the ETX cost to node $E$ from each node in the network of Fig. 1(a). Each node's ETX cost is the sum of the link ETX cost along the lowest-ETX path to destination $E$. The ETX cost of a link is the inverse of the link's delivery probability [9]. For instance, $C$ 's ETX cost to $E$ is the sum of the ETX of link $C-D(1.11)$ and the ETX of link $D-E(1.11)$. Naturally, the source chooses the path to the destination with the lowest ETX cost. If source node $A$ is running LQSR, it will select node $D$ 
as the next-hop relay node in the path $A-D-E$. Also, if $A$ is running ExOR, it will regard the node $D$ as the candidate with the highest priority in the forwarder list [15].

It should be noted that the above calculation is carried out without concern about the delivery rate of CR links, i.e. the dashed links. When the primary user is "ON", the delivery rate of the $\mathrm{CR}$ link is definitely zero since the CR users cannot cause interference to the licensed users [3]. But when the primary user is "OFF", the CR link is available for packet delivery. So the CR link can be considered as a two state link. For example, the delivery rate of link $C-D(0.95,0)$ is 0.95 when the primary user is "OFF" but 0 when the primary user is "ON".

Taking the CR links into account, let us recheck the problem of optimal forwarder selection and compute delivery probabilities of different paths. The probability of a packet being successfully delivered to the destination when sending via node $D$ is $p_{A D} * p_{D E}=0.36$ and the cost is 3.61. Correspondingly, the possibility of a packet being successfully delivered when going through node $B$ is $p_{A B} * p_{B D} * p_{D E}=0.32$ and the cost is 4.72 . In the same way, we can obtain the corresponding values via node $C$. There is no doubt that the ETX based criteria would make us select node $D$ as the optimal forwarder from the source since the other nodes have lower delivery probability and larger cost to destination than $D$. However, when the primary user is not present, $B$ has another CR link to relay packets to $D$, and the delivery fails only when the two links between $B$ and $D$ are both down. Therefore, the probability of delivery via $B$ should be computed as $p_{A B} *\left(1-\left(1-p_{B D}\right) *\left(1-p_{B D}^{c r}\right)\right) * p_{D E}=$ $0.9 *(1-(1-0.4) *(1-0.7)) * 0.9=0.66$, and the cost is 3.42 . Thus, $B$ should be the forwarder with the highest priority when the primary user is "OFF", and "D" should the the forwarder with the highest priority when the primary user is "ON".

If the prioritization of candidates for $\mathrm{OR}$ in $\mathrm{CR}$ networks is only driven by ETX, qualified candidates would be ignored, and as a result the source would make a routing decision that provides a significantly lower delivery probability.

\section{SYSTEM MODEL}

\section{A. OR in the CR Network}

We consider a multi-hop CR network with only one primary user and $N \mathrm{CR}$ nodes arbitrarily located on a plane. The $N$ CR users are able to operate on the licensed bands only if the primary user is not present. When the primary user returns, all the $\mathrm{CR}$ nodes have to vacate the licensed bands as soon as possible. The effective transmission range over different frequencies should not be the same. If the CR nodes are using the vacant bands of the primary user, the $\mathrm{CR}$ transmission range is defined to be $R_{c r}$. Otherwise, the transmission range is defined as $R$.

Assume node $n_{i}(1 \leq i \leq N)$ is forwarding a packet to the destination node. Each node can transmit the packet at the same rate $R$. A usable link $l_{i j}$ from node $n_{i}$ to $n_{j}$ is acknowledged, when the packet delivery ratio, denoted as $p_{i j}$, from $n_{i}$ to $n_{j}$ is larger than 0.1 [14], [15]. We denote the set of nodes within the effective transmission range of node $n_{i}$ as the neighboring node set $J_{i}$ of node $n_{i}$. Note that, for different effective transmission ranges, neighboring node sets of node $n_{i}$ are different, and so it is with the packet delivery ratio on the same link [8], [11]. We define the set $F_{i}:=\left\langle n_{i_{1}}, n_{i_{2}} \ldots n_{i_{r}}\right\rangle$, as forwarding candidate set, which is a subset of $J_{i}$ and includes all the nodes selected to be involved in the opportunistic forwarding based on some particular selection strategies [8]. $F_{i}$ is an ordered set of nodes, where the order represents the priority in forwarding a received packet.

The OR works as follows: the source node $n_{s}$ is supposed to have a complete knowledge of the packet delivery ratio of all the nodes in the networks by using a link-state flooding scheme periodically, where the typical time is defined as $T$. $n_{s}$ selects $F_{s}$ from $J_{i}$; then broadcasts the packet to the nodes in its forwarding candidate set $F_{s}$. Candidates in $F$ follow a specific priority to relay the packet, i.e., a forwarding candidate will forward the message only when all the nodes with higher priorities fail to do so. When no forwarding candidate has successfully received the packet, the sender will retransmit the packet if retransmission is enabled. The sender will drop 
the packet when the number of retransmissions exceeds the limit. The forwarding reiterates until the packet is delivered to the destination.

\section{B. Related CR Models}

1) Activities of the Primary User: We assume that primary user activities can be modeled as exponentially distributed inter-arrivals [17]. In this model, the primary user traffic can be simplified as a two state birth-death process with birth rate $\alpha$ and death rate $\beta$. An "ON" state represents the period used by primary users and an "OFF" state represents the unused period. Since each user arrival is independent, each transition follows the Poisson arrival process. Thus, the length of "ON" and "OFF" periods are exponentially distributed.

From the primary user activity model, we can estimate the posteriori probabilities as the following [18],

$$
\begin{aligned}
& P_{\text {on }}=\frac{\alpha}{\alpha+\beta} \\
& P_{\text {off }}=\frac{\beta}{\alpha+\beta}
\end{aligned}
$$

where $P_{o n}$ is the probability of the time used by primary user (i.e. primary user is "ON") and $P_{o f f}$ is the probability of the idle period (i.e. primary user is "OFF").

2) Detection Probability of the CR Nodes: Suppose the CR node set within the transmission range $R_{c r}$ of $n_{i}$ can be denoted as $M_{i}$, and the individual detection probability and false alarm probability ${ }^{2}$ of nodes in $M_{i}$ are $p_{d_{i}^{j}}$ and $p_{f_{i}^{j}} j \in M_{i}$.

In order to mitigate the multi-path fading and shadowing effects of the individual detection, cooperative detection among CR users are performed as proposed in [19], [20]. Probabilities of detection and false alarm for the cooperative scheme (denoted by $Q_{d_{i}}$ and $Q_{f_{i}}$ respectively) can be written as,

$$
\begin{aligned}
& Q_{d_{i}}=1-\prod_{j \in M_{i}}\left(1-P_{d_{i}}^{j}\right) \\
& Q_{f_{i}}=1-\prod_{j \in M_{i}}\left(1-P_{f_{i}}^{j}\right)
\end{aligned}
$$

From the two equations above, it may be seen that compared with individual sensing, cooperation schemes

\footnotetext{
${ }^{2}$ The calculation of $p_{d}$ and $p_{f}$ relies on different parameters when different detection methods are exploited. See details in [3], [23-25]
}

increase the probability of detection as well as probability of false alarm.

\section{Details of the Cost Design}

This section describes our cost design for OR in the CR network. The proposed metric incorporates the characters of the CR into the link cost, and leads to the optimal OR forwarder selection.

\section{A. Link Cost}

We define the link cost $c_{i j}(i)$ as the cost to send a packet from the node $n_{i}$ to the node $n_{j}$ along the link $l_{i j}$ in CR network. Without loss of generality, we assume there are both traditional link $l_{i j_{t r}}$ and CR link $l_{i j_{c r}}$ between the two nodes.

Since the CR link is not always feasible, the packet delivery ratio of $l_{i j}$ should be piece-wise over time. When the primary user is "ON", the delivery ratio of $l_{i j}$ is simply equal to that of the traditional link $l_{i j_{t r}}$. Thus, delivery ratio during the "ON" period $p_{i j_{o n}}$ is,

$$
p_{i j_{o n}}=p_{i j}^{t r}
$$

On the contrary, when the primary user is "OFF", $p_{i j_{\text {off }}}$ becomes the probability that a packet from $n_{i}$ is forwarded to $n_{j}$ either by the traditional link $l_{i j_{t r}}$ or by the CR link $l_{i j_{t r}}$. As a result, the delivery ratio $p_{i j_{o f f}}$ can be expressed as,

$$
p_{i j_{o f f}}=1-\left(1-p_{i j}^{t r}\right)\left(1-p_{i j}^{c r}\right)
$$

Now, we generalize the proposed concept of the link cost in CR network as,

Definition. Let $l_{i j}$ be the link from node $n_{i}$ to $n_{j}$ in the $C R$ network. The cost of $l_{i j}$, denoted by $c_{i j}$, is the the expected number of transmissions jointly considering the existence of traditional link and CR link over the period of link-state flooding test $T^{3}$ :

$$
c_{i j}(i)=\frac{T_{o n_{i}} \cdot \frac{1}{p_{i j_{o n}}}+T_{o f f_{i}} \cdot \frac{1}{p_{i j_{o f f}}}}{T} .
$$

where $T_{o n_{i}}$ is the time period when the CR link with source $n_{i}$ is valid, and $T_{o f f_{i}}$ corresponds to the time when the CR link is not available.

\footnotetext{
${ }^{3}$ The typical period of link-state flooding is 20 minutes [14]
} 
It can be deduced from the definition that the link cost between any pair of nodes in CR network is closely related to the valid time of their CR link.

\section{B. Valid Time of the CR Link}

Since the CR users cannot communicate with the primary users or cause any interference to licensed usage, the valid time of the CR links depends on both activities of the primary user and the detection performance of the CR users. For source node $n_{i}$, the valid time of the CR link should be expressed as,

$$
\begin{gathered}
T_{o n_{i}}=T-T_{o f f_{i}}-T \cdot P_{o n} \cdot\left(1-Q_{d_{i}}\right) \\
T_{o f f_{i}}=T \cdot\left(P_{o n} \cdot Q_{d_{i}}+P_{o f f} \cdot Q_{f_{i}}\right)
\end{gathered}
$$

where both $T_{o f f_{i}}$ and $T_{o n_{i}}$ are based on the cooperative detection of the CR nodes in $C_{i}$ mentioned in Sec. IIIB-(2). To be specific, $T_{o f f_{i}}$ consists of two parts: when the primary user is "ON" and $\mathrm{CR}$ nodes in $C_{i}$ have detected his presence, the CR links are not valid; when the primary user is "OFF", CR nodes in $C_{i}$ send false alarms, which results in the pending of delivery over these CR links. Similar analysis is also applicable to $T_{o n_{i}}$. Note that $\left(1-Q_{d_{i}}\right)$ is the probability of missing detecting the primary user. $T \cdot P_{o n} \cdot\left(1-Q_{d_{i}}\right)$ is deducted from $T-T_{o f f_{i}}$ because when the primary user returns, all the CR nodes must evacuate the frequency bands unconditionally.

\section{Candidate Forwarders Prioritization}

The prioritization of candidate forwarders play an important role in the performance of OR in CR network, because it affects both the number of transmissions and the throughput. Take the throughput as an example, if a candidate with poor link quality from the sender but a high priority in the forwarder list, in most of times, it will not receive the packet correctly. According to the mechanism of OR, lower-priority candidates always have to wait for a period of time to confirm his failure before they are likely to relay the packet, which in result increases the total medium time and decreases the throughput.

Therefore, the prioritization of candidate forwarders should satisfy the following requirements:
- It is based on delivery ratios, which directly affect throughput.

- It penalizes routes with more hops, which have lower throughput due to interference between different hops of the same path.

- It considers number of transmissions.

As to OR in CR network, source $n_{i}$ prioritizes the candidate $n_{j} \in J_{i}$ based on the expected cost of delivering a packet to the destination. Since there are several possible paths going through $n_{j}$ to the destination, we now define the cost of a path as,

Definition. Let $V=\left(n_{1}, n_{2}, \ldots n_{k}\right)$ be a path from the source $n_{1}$ to the destination $n_{k}$ in $\Re$, where $\Re$ is the set of possible paths. The cost of $V$, denoted $C(V)$, is the sum of the link costs of the nodes in $V$ :

$$
C(V)=c_{12}(1)+c_{23}(2)+\ldots c_{(k-1) k}\left(n_{k-1}\right) .
$$

To specifies the forwarder list in priority order, $n_{i}$ needs to calculate the cost of $V_{j} \in \Re_{j}$ for node $n_{j}$, finds the lowest path through $n_{j}$, and compares it with other nodes in $J_{i}$.

\section{Evaluation AND ANALysis}

In this section, a simulation environment is developed modeling the operation of OR in a multi-hop CR network with the proposed cost design (i.e. CROR). In order to determine the effectiveness of CROR, the performance of CROR based routing is compared with that of ETX based OR (i.e. ExOR) and ETX based single-path routing (i.e. LQSR). The evaluation metrics are number of transmissions and end-to-end throughput.

\section{A. Simulation Setup}

The simulation is created to model a CR network containing a potential primary user and $50 \mathrm{CR}$ nodes randomly distributed on a $1000 \mathrm{~m} \times 1000 \mathrm{~m}$ plane, as displayed in Fig. 2(a) and (b). The birth rate $\alpha$ of the primary user is set to be 0.3 , and the death rate $\beta$ is 0.4 . Every CR node is granted to use the frequency bands of $802.11 \mathrm{~b}$, and has the ability to dynamically access to the licensed frequency bands of $802.11 \mathrm{~g}$ if the primary user is "OFF". The effective transmission radii of $802.11 \mathrm{~b}$ and $802.11 \mathrm{~g}$ are $304 \mathrm{~m}$ and $183 \mathrm{~m}$ [22], respectively. 
TABLE I

Simulation Parameters

\begin{tabular}{|c|c|c|}
\hline \hline Parameter & \multicolumn{2}{|c|}{ Value } \\
\hline CR network & $802.11 \mathrm{~b}$ & $802.11 \mathrm{~g}$ \\
\hline Bit Rate (BR) & $11 \mathrm{Mbps}$ & $11 \mathrm{Mbps}$ \\
\hline Transmission Range & $R=304 m$ & $R_{c r}=183 m$ \\
\hline Basic Bit Rate (BBR) & \multicolumn{2}{|c|}{$1 \mathrm{Mbps}$} \\
\hline PHY Header Size (PHS) & \multicolumn{2}{|c|}{$192 \mathrm{bits}$} \\
\hline MAC Header Size (MHS) & \multicolumn{2}{|c|}{$272 \mathrm{bits}$} \\
\hline$T_{h}$ & PHS/BBR + MHS/BR \\
\hline$T_{A C K}$ & $112 / \mathrm{BR}+\mathrm{PHS} / \mathrm{BBR}$ \\
\hline$T_{S I F S}$ & \multicolumn{2}{|c|}{$10 \mu \mathrm{s}$} \\
\hline$T_{D I F S}$ & \multicolumn{2}{|c}{$50 \mu \mathrm{s}$} \\
\hline \hline
\end{tabular}

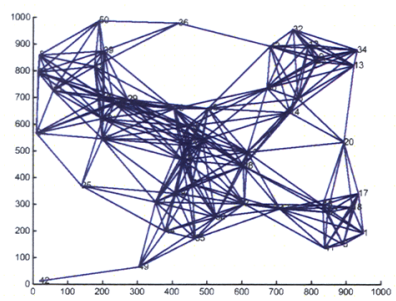

(a) Traditional links between node pairs with $802.11 \mathrm{a}$

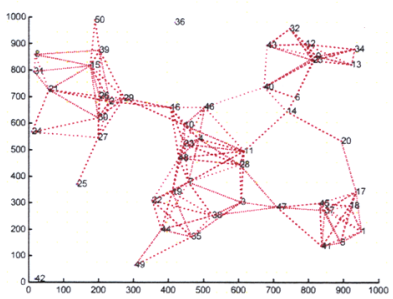
with $802.11 \mathrm{~b}$ (b) CR links between node pairs

Fig. 2. Physical layout of the 50 nodes participating in the simulations

The delivery ratio between two nodes is based on the distance-to-delivery relationship [21], [14] and [8], where the packet delivery ratio is inversely proportional to the distance with random gaussian deviation of 0.1 . Opportunistic routes are chosen using a candidate set size of 4 . The data payload size is 512 bytes, and the medium time cost of OR can be calculated using the other parameters listed in Table I as illustrated in [11].

In this simulation, no medium contention or any radio propagation effects are considered except the random packet loss based on the delivery ratios. In addition, all the nodes are assumed to be stationary throughout the simulation.

\section{B. Results and Analysis}

Fig. 3 compares the average number of transmissions required to route 200 packets between every pair of nodes in the CR network. The results indicate CROR and ExOR outperform LQSR often by a margin of $50 \%$ to $65 \%$, which agrees with the results in [14]. The reason

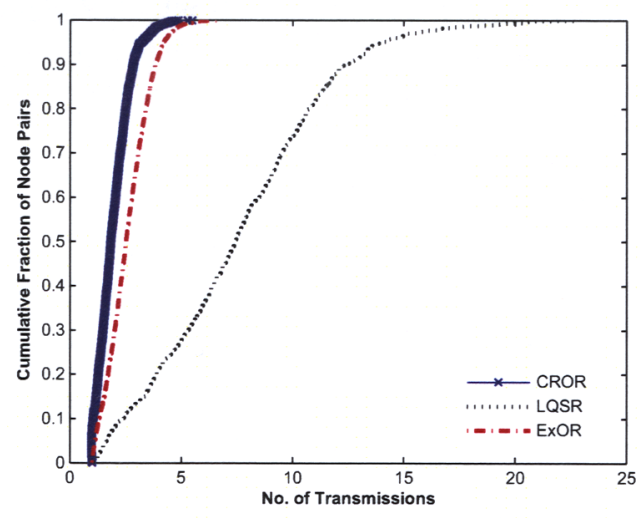

Fig. 3. CDF of number of transmissions required to route a packet from source to destination.

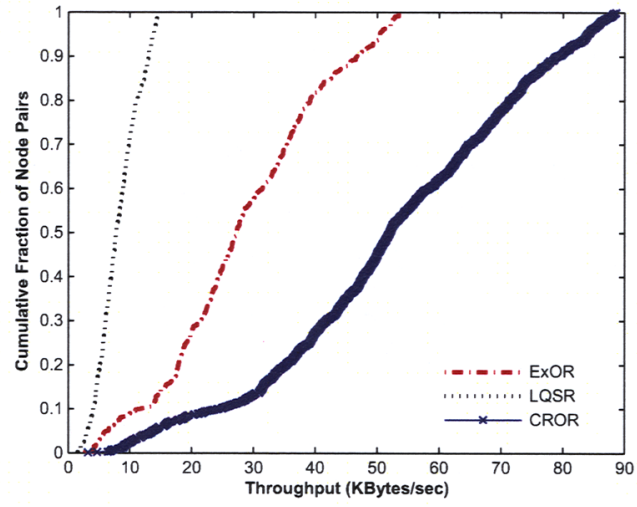

Fig. 4. CDF of end-to-end throughput.

is that CROR and ExOR choose routes opportunistically and have a bunch of opportunities to skip intermediate hops, which LQSR is not able to overlook. The CROR approach is better than ExOR because the delivery ratios may increase when there are both traditional link and CR link between the node pairs.

Correspondingly, Fig. 4 compares the throughput CDFs of CROR, ExOR and LQSR for all the $50 \times 49$ node pairs. CROR's throughput achieves $60 \mathrm{KBytes} / \mathrm{sec}$ for the median pair, whereas ExOR's throughput is 33 KBytes/sec for the median pair, and LQSR is only 11 KBytes/sec for the median pair. Due to the considerations of CR links, optimal candidate forwarder is selected, and waiting time of the other nodes in the forwarder list is saved in CROR based OR, which incurs 
the trunking gain of the end-to-end throughput.

\section{CONCLUSION}

The cost design of a routing protocol must derive from the characteristics of its target networks. In this paper, we design a cost criterion for OR in CR network to specify the candidate forwarder list in order. This criterion takes both the time varying features of the CR links and the activities of the primary user into consideration. Evaluation and analysis show that the proposed cost criterion leads to the optimal selection of forwarder among relay candidates, and CROR based OR effectively increases end-to-end throughput of the node pairs and decreases the number of transmissions compared with existing ETX based OR as well as LQSR.

\section{REFERENCES}

[1] Federal Communications Commision, "Spectrum policy task force report," Report, Et docket No. 02-135, Nov. 2002.

[2] J. Yang, Spatial channel characterization for cognitive radios, $M S$ Thesis, UC Berkeley, 2004.

[3] I. F. Akyildiz, W. Lee, M. Vuran, and M. Shantidev, "NeXt generation/ dynamic spectrum access/ cognitive radio wireless networks: a survey", Computer Networks Journal (Elsevier), vol. 50, pp.2127-2159, Sep. 2006.

[4] X. Liu and S. Shankar, "Sensing-based opportunistic channel access", ACM Journal on Mobile Networks and Applications (MONET), vol. 11, pp. 577-591, Feb. 2006.

[5] H. Kim and K. Shin, "Efficient Discovery of Spectrum Opportunities with MAC-Layer Sensing in Cognitive Radio Networks", IEEE Transactions on Mobile Computing, vol. 7, No. 5, pp. 533545, May 2008.

[6] Joint Tactical Radio System, http://enterprise.spawar.navy.mil/.

[7] SAFECOM ł http://www.safecomprogram.gov/SAFECOM/.

[8] K. Zeng, W. Lou, and H. Zhai, "On End-to-end Throughput of Opportunistic Routing in Multirate and Multihop Wireless Networks," In Proc. of INFOCOM 08', pp. 816-824, Apr. 2008.

[9] D. Couto, D. Aguayo, J. Bicket, and R. Morris, "A highthroughput path metric for multi-hop wireless routing," In Proc. of ACM/IEEE MobiCom, Sep. 2003.

[10] J. Zhao, R. Govindan, "Understanding packet delivery performance in dense wireless sensor networks," In Proc. of ACM Sensys03, Los Angeles, Nov. 2003.

[11] K. Zeng, W. Lou, J. Yang, D. Brown, "On throughput efficiency of geographic opportunistic routing in multihop wireless networks," In Proc. of QShine07, Vancouver, Aug. 2007.

[12] H. Fussler, J. Widmer, M. Kasemann, M. Mauve, and H. Hartenstein, "Contention-based forwarding for mobile ad-hoc networks," Elseviers Ad Hoc Network, vol. 1 pp. 351-369, Nov. 2003.
[13] M. Zorzi, R. Rao, "Geographic random forwarding (geraf) for ad hoc and sensor networks: energy and latency performance," IEEE Trans Mobile Computing, vol. 2 pp. 349C365, 2004.

[14] S. Biswas, and R. Morris, "Opportunistic Routing in MultiHop Wireless Networks," In Proc. of the ACM Symposium on Communications Architectures and Protocols, SIGCOMM 05', Philadelphia, 2005.

[15] S. Biswas, and R. Morris, "Exor: opportunistic multihop routing for wireless networks," In Proc. of SIGCOMM05, Aug. 2005.

[16] D. Richard, P. Jitendra, and Z. Brian, "Routing in Multi- Radio, Multi-Hop Wireless Mesh Networks," In Proc. of ACM Mobicom, 2004.

[17] K. Sriram, and W. Whitt, "Characterizing Superposition Arrival Processes in Packet Multiplexers for Voice and Data," IEEE Journal on Selected Areas in Communications, vol. SAC-4, pp. 833-846, Sep. 1986.

[18] W. Lee, I. F. Akyildiz, "Optimal Spectrum Sensing Framework for Cognitive Radio Networks," to appear in IEEE Transaction on Wireless Communications, 2008.

[19] J. Hillenbrand, T. Weiss and F. Jondral, "Calculation of detection and false alarm probabilities in spectrum pooling systems," IEEE Communications Letters, vol. 9, no. 4, pp. 349C351, April 2005.

[20] S. Haykin, "Cognitive Radio: Brain-Empowered Wireless Communications," IEEE Journal on Selected Areas in Communications, vol. 23, no. 2, pp. 201C220, Feb. 2005.

[21] D. Ganesan, B. Krishnamachari, A. Woo, D. Culler, D. Estrin, and S. Wicker, "Complex behavior at scale: An experimental study of low-power wireless sensor networks," Technical report UCLA/CSD-TR 02-0013, UCLA CS Department, 2002.

[22] D. Cabric, S.M. Mishra, and R.W. Brodersen, "Implementation Issues in Spectrum Sensing for Cognitive Radios," in Proc. of Asilomar Conference on Signals, Systems, and Computers, pp. 772-776, Nov. 2004.

[23] H. Tang, "Some Physical Layer Issues of Wide-Band Cognitive Radio Systems," in Proc. IEEE International Symp. Dynamic Spectrum Access Networks, DySPAN '05, pp. 151-159, Nov. 2005.

[24] S. Shankar, C. Cordeiro, and K. Challapali, "Spectrum Agile Radios: Utilization and Sensing Architectures," in Proc. IEEE International Symp. Dynamic Spectrum Access Networks, DySPAN '05, pp. 160- 169, Nov. 2005.

[25] Cisco Aironet 802.11a/b/g Wireless LAN Client Adapters (CB21AG and PI21AG) Installation and Configuration Guide, Cisco Systems, Inc., 2004. 